\title{
Administración y preparación de antimicrobianos en una unidad chilena de cuidados neonatales
}

\author{
Preparation and dispensing of antimicrobials \\ in a Chilean neonatal care unit
}

\section{Administração e preparação de antimicrobianos em uma unidade chilena de cuidados neonatais}

\author{
F. Cruz ${ }^{\mathrm{a}}$, P. Gálvez ${ }^{\mathrm{b}{ }^{*}}$ \\ ORCID: \\ ${ }^{a}$ https://orcid.org/o0oo-0o01-6439-7412 \\ ${ }^{\mathrm{b}} \mathrm{https}$ ///orcid.org/0000-0002-4847-5692 \\ 'Universidad de Chile, Facultad de Medicina, Escuela de Obstetricia, Santiago, Chile \\ Universidad de Chile, Facultad de Medicina, Departamento de Promoción de la Salud de la Mujer y el Recién \\ Nacido, Santiago, Chile
}

Recibido: 05 mayo 2019 / Aceptado: 30 noviembre 2019

\begin{abstract}
RESUMEN
Objetivo: Describir la preparación, administración y monitorización de los antimicrobianos utilizados en una Unidad de Cuidados Neonatales en Santiago de Chile.

Métodos: Estudio descriptivo. Se realizó la aplicación de un instrumento a 20 matronas de neonatología de una Unidad Chilena de Cuidados Neonatales (UCHCN) en 2018, para recopilar información respecto a once variables en la preparación y administración de antimicrobianos. El estudio abarcó tres variables principales: preparación, mantención y monitorización de los antimicrobianos.

Resultados: Se recopiló información de 14 antimicrobianos, de los cuales la presentación del 92.8\% de ellos era inyectable y el $78.2 \%$ de los encuestados reconocía el valor de la unidad de dosificación del medicamento. Mantención 85.7\% de los antimicrobianos son eliminados luego de su preparación. Para dilución se utiliza mayormente la solución fisiológica en un $76.9 \%$ de los casos y el volumen utilizado de diluyente y concentración final varió según cada medicamento. Un $92.8 \%$ de los antimicrobianos se administra por vía endovenosa y todos a través de una bomba de jeringa. Monitorización específica de la administración sólo se realiza en un $7.2 \%$ de ellos.
\end{abstract}

\footnotetext{
*Autor para correspondencia. Correo electrónico: pagalvezo@uchile.cl https://doi.org/10.22201/eneo.23958421e.2020.1.619

1665-7063/@ 2020 Universidad Nacional Autónoma de México, Escuela Nacional de Enfermería y Obstetricia. Este es un artículo Open Access bajo la licencia CC BY-NC-ND (http://creativecommons.org/licenses/by-nc-nd/4.o/).
} 
Conclusiones: Debido a las variabilidades encontradas en los procesos de mantención, dilución y monitorización es que se propone la creación o el mejoramiento y difusión de protocolos locales, para evitar efectos adversos o complicaciones y así resguardar la seguridad de los pacientes. Palabras clave: Antimicrobianos; neonatología; monitoreo de medicamentos; Chile.

\section{ABSTRACT}

Objetive: To describe the preparation, dispensing, and monitoring of antimicrobials used in a Neonatal Care Unit in Santiago de Chile.

Methods: This is a descriptive study. An assessment instrument was administered to 20 midwives laboring in a Chilean Neonatal Care Unit during 2018 in order to gather information on 11 variables related to the preparation, dispensing, and monitoring of antimicrobials. Results: Information was gathered on 14 antimicrobials; $92.8 \%$ of these were injectable. $78.2 \%$ of the surveyed personnel recognized the value of the drug dosification unit. $85.7 \%$ of antimicrobials were eliminated after their preparation. Regarding the dilution, in $76.9 \%$ of the cases saline solution was used with diverse concentrations. A close monitoring of the drug administration was followed only in $7.2 \%$ of the cases.

Conclusions: Due to the variability found in the processes of preservation, dilution, and monitoring of antimicrobials in this Neonatal Care Unit, an implementation of a local protocol to improve the procedures and avoid adverse events or complications is proposed.

Keywords: Antimicrobials; neonatology; drug monitoring; Chile.

\section{RESUMO}

Objetivo: Descrever a preparação, administração e monitorização dos antimicrobianos utilizados em uma Unidade de Cuidados Neonatais em Santiago de Chile.

Métodos: Estudo descritivo. Realizou-se a aplicação de um instrumento a 20 parteiras de neonatologia de uma Unidade Chilena de Cuidados Neonatais (UCHCN) em 2018, para recopilar informação ao respeito de onze variáveis na preparação e administração de antimicrobianos. O estudo abrangeu três variáveis principais: preparação, manutenção e monitorização dos antimicrobianos.

Resultados: Recopilou-se informação de 14 antimicrobianos, dos quais a apresentação do 92.8\% deles era injetável e o 78.2\% dos inquiridos reconhecia o valor da unidade de dosagem do medicamento. Manutenção $85.7 \%$ dos antimicrobianos são eliminados depois de sua preparação. Para diluição utiliza-se maiormente a solução fisiológica em um 76.9\% dos casos e o volume utilizado de diluente e concentração final variou segundo cada medicamento. Um 92.8\% dos antimicrobianos administra-se por via endovenosa e todos através de uma bomba de seringa. Monitoramento específica da administração só se realiza em um 7.2\% deles. Conclusões: Devido às variabilidades encontradas nos processos de manutenção, diluição e monitoramento, é que se propõe a criação ou a melhoria e difusão de protocolos locais, para evitar efeitos adversos ou complicações e assim resguardar a segurança dos pacientes.

Palavras chave: Antimicrobianos; neonatologia; monitoramento de medicamentos; Chile.

\section{INTRODUCCIÓN}

La mayoría de los neonatos que son ingresados y hospitalizados en las unidades de neonatología, corresponden a recién nacidos prematuros que requieren de un diagnóstico y tratamiento oportuno para las complicaciones que presentan o, la prevención de éstas, garantizando estándares óptimos 
de calidad, seguridad y eficiencia al prestar el mejor servicio en el cuidado de ellos ${ }^{1}$. Debido a esto, se genera una gran demanda de atención médica de alta complejidad, como lo expresado en las guías chilenas de neonatología, las cuales destacan como funciones: exámenes y procedimientos diagnósticos, cuidados intensivos, intervenciones quirúrgicas y tratamientos farmacológicos costosos y prolongados, todo lo cual requiere una adecuada disponibilidad de recursos (...) que garanticen una atención oportuna y de buena calidad...2.

A partir de este escenario es que cobra gran importancia el uso de fármacos en las unidades de neonatología, obteniéndose grandes beneficios gracias al acceso que se tiene a estos, la calidad y a su uso racional ${ }^{3}$. Sin embargo, para una administración segura y eficaz de medicamentos en unidades neonatales, se deben considerar aspectos de la fisiología neonatal, la farmacocinética neonatal (que incluye procesos de absorción, distribución, metabolismo y excreción que difieren de otras poblaciones) $)^{3,5}$ y farmacodinamia de los medicamentos a administrar, para lograr los objetivos terapéuticos con los menores riesgos. En cuanto a esto, se describe a la fisiología neonatal como un sistema de grandes cambios y de maduración, donde hay diferencias en la composición corporal, flujo sanguíneo, tamaño de los órganos o concentraciones de proteínas plasmáticas ${ }^{4,6}$, a diferencia de otro tipo de personas como pediátricos o adultos, en los que se centra principalmente la investigación farmacológica ${ }^{5,6}$. Además de lo anterior, en estos pacientes existen procesos patológicos propios de la inmadurez o relacionados con la gestación, lo que lleva a gran variabilidad en los efectos que puede tener un fármaco 4 , por lo que es necesario evaluar todos estos aspectos de la farmacología neonatal, y evitar la extrapolación de dosis pediátricas o de adultos a neonatos ${ }^{5,7}$.

A nivel internacional existe escasa literatura publicada en bases informáticas reconocidas por la comunidad científica sobre preparación y administración de antimicrobianos en neonatología. Una de las causas de esto es el hecho de que aún no se ha estudiado adecuadamente la farmacocinética en la población neonatal4, debido a la falta de ensayos clínicos de investigación pediátrica por aspectos éticos-legales ${ }^{8,9}$. Sumado a esto, los protocolos o documentos científicos que desarrollan la temática, requieren de un pago adicional para obtenerlos o están publicados en un idioma que no es el español, como es el caso de la principal referencia local, Neofax ${ }^{\circledR}$ de Micromedex $^{10}$.

En Chile, el hospital de estudio ha elaborado protocolos de administración de antimicrobianos endovenosos en pacientes adultos, pediátricos y neonatales ${ }^{11}$, el cual menciona de forma general la preparación de medicamentos, indica materiales a utilizar, cálculos de dosis y algunas consideraciones especiales. El protocolo de almacenamiento y conservación de medicamentos ${ }^{12}$, menciona reglas generales, como temperatura, lugar y condiciones de conservación, señala en la mayoría de las familias de fármacos que se debe guiar por lo estipulado por el fabricante, por lo que no es utilizado para estandarizar. A nivel de la Unidad de Neonatología, se ha creado una guía para la preparación y administración segura de medicamentos que pretende unificar criterios y estandarizar procesos de dilución, concentración, compatibilidades y estabilidad de ciertos medicamentos; sin embargo, este último, no es conocido por todos los profesionales que allí trabajan.

Por lo anteriormente descrito, se hace fundamental la farmacología clínica y la vigilancia que se realiza a los neonatos cuando se les prescribe un medicamento, ya que, a pesar de ser ampliamente utilizados en estas unidades, en Chile y en muchos países latinoamericanos no existen normativas locales universales que regulen la preparación, mantención, y vigilancia (o monitorización) de los fármacos empleados en dichas unidades. Esta ausencia de protocolización en el uso de fármacos puede poner en riesgo la seguridad de los recién nacidos, deja a criterio clínico la utilización de los mismos, a pesar de que actualmente existe evidencia suficiente que demuestra la eficacia de la estandarización. 
La variable preparación se definió como el proceso de manipulación del medicamento a través de diferentes pasos para lograr una concentración y dosis adecuada para ser administrada, desde la recolección de los materiales, el cálculo de la dosis y su dilución. La variable mantención corresponde al intervalo de tiempo durante el cual se espera que un producto medicamentoso, si se almacena correctamente, satisfaga las especificaciones establecidas. Finalmente, la variable vigilancia se definió como la Identificación y valoración de los efectos del uso, agudo y crónico de los tratamientos farmacológicos.

Por todo lo mencionado, es que se realiza esta investigación cuyo objetivo general es describir la preparación, administración y monitorización de antimicrobianos que realizan matronas y matrones de neonatología de un UCHCN de Santiago, con base en su experiencia y conocimientos, a través de la identificación y determinación de la dilución, la mantención y monitorización, y junto a esto comparar los resultados con lo establecido en su normativa local y recomendaciones internacionales, con el propósito de recomendar criterios de preparación, mantención y administración de antimicrobianos en neonatología a través de protocolos claros, conocidos y accesibles, que estandaricen estos procedimientos para disminuir posibles errores y sus consecuencias.

\section{MÉTODOS}

Este estudio de diseño cuantitativo, observacional, transversal y de tipo descriptivo, se llevó a cabo en la Unidad de Neonatología de un centro hospitalario de Santiago, Chile en 2018. Se aplicó un instrumento tipo cuestionario a los profesionales matrona o matrón, que estén encargados de la preparación y/o administración de antimicrobianos en la Unidad. El instrumento es de confección propia y fue elaborado en 2017, el cual fue previamente validado semánticamente por 8 académicos de neonatología del Departamento de Promoción de la Salud de la Mujer y el Recién Nacido de la Universidad de Chile. El cuestionario recoge información respecto a once variables en cuanto a la preparación y administración de 14 antimicrobianos, los cuales fueron elegidos por ser los más frecuentemente utilizados en la unidad (amikacina, ampicilina, anfotericina B, cefazolina, cefotaxima, cloxacilina, eritromicina, centamicina, linezolid, meropenem, metronidazol, penicilina, piperacilina/tazobactam y vancomicina).

Los criterios de inclusión son los siguientes: empleada/o actual del centro hospitalario a estudiar, pertenecer a la unidad de neonatología y estar encargada/o de la preparación y/o administración de antimicrobianos. No se plantearon criterios de exclusión. El universo para abordar constaba de un total de 28 matronas y matrones de la unidad de neonatología.

Las variables solicitadas por cada antimicrobiano fueron: presentación del fármaco (forma física que caracteriza al producto farmacéutico: inyectable, cápsulas, jarabe, entre otros, y su unidad de dosificación); tiempo de mantención tras la preparación (se midió en días); lugar de mantención del preparado (refrigerador o ambiente); dilución del fármaco (adición de diluyente para conseguir una osmolaridad final lo más parecida a la sanguínea: solución fisiológica, agua bidestilada, solución glucosada al 5\%, u otro); volumen del diluyente (en mililitros); concentración final del fármaco diluido (valor numérico y unidad de medida de dosificación); vía de administración (endovenosa, oral, intramuscular, subcutánea u otras) y tiempo de administración (en minutos u horas); método de administración (utilización y tipo de dispositivo electrónico para la administración controlada de sustancias por vía endovenosa: bomba jeringa o casete), y monitorización (identificación y valoración de los efectos del uso de los antimicrobianos a través de signos clínicos o análisis de laboratorio). 
La investigadora principal aplicó el instrumento a los profesionales, siendo la única encuestadora para reducir la variabilidad en proceso. Previamente se realizó entrega y firma de un consentimiento informado a todos los participantes.

El análisis de los datos se realizó a través de frecuencias absolutas y relativas, y son presentadas en tablas de distribución de frecuencias. Los resultados de la recolección de datos fueron tabulados en Microsoft Excel ${ }^{\circledR}$ y analizados con el Software estadístico Stata $13.0^{\circledR}$.

Este protocolo fue aprobado por el comité científico del Hospital San Juan de Dios, Santiago de Chile y el comité de ética de investigación en seres humanos de la Facultad de Medicina de la Universidad de Chile.

\section{RESULTADOS} ción y administración de antimicrobianos, de los cuales 4 no cumplían con los criterios de selección y 4 no quisieron participar, por lo que 20 fueron considerados. Un 90\% de los participantes fueron mujeres.

En cuanto a la variable presentación del fármaco en un 92.8\% fue inyectable y 7.2\% jarabe, esto tiene relación con el hecho de que la mayoría de los antimicrobianos en las unidades de neonatología son administrados por vía parenteral. Un 78.5\% de los encuestados reconocía el valor de la unidad de dosificación de los antimicrobianos, es decir el gramaje u otro, cuando se les consultaba al respecto (Tabla 1).

Respecto al proceso de dilución de los antimicrobianos inyectables, se preguntaron por tres variables, diluyente utilizado, que en un 76.9\% de los antimicrobianos fue solución fisiológica, seguido de agua bidestilada. Los valores de volumen utilizado de diluyente y concentración final del fármaco dependen de la concentración final máxima de cada uno, pero van desde 5 a $60 \mathrm{ml}$ (Tabla 1). La vía de administración de los antimicrobianos también es la misma, con una concordancia del 100\%.

Al preguntar por el tiempo de mantención de los antimicrobianos se aprecia que un $85.7 \%$ (12 de ellos) son eliminados una vez preparados y un 7.1\% (Vancomicina) se mantiene máximo por un día. Con relación al lugar de mantención de los antimicrobianos que pueden reservarse, un 100\% de los encuestados utiliza el refrigerador de la unidad, el cual es destinado exclusivamente para este fin y acorde a las recomendaciones locales.

Referente a la administración, se preguntó por la vía, el tiempo y método. Frente a esto, se observó que un 92.8\% de los antimicrobianos se ministran por vía endovenosa. En un lapso aproximado de una hora (61.5\%) mayoritariamente 1 hora, con un 61.5\%. Esto es correlativo con lo que menciona la literatura internaciona ${ }^{10} \mathrm{y}$ local, que recomienda un tiempo de infusión entre 30 minutos a 1 hora, manteniéndose los mismos porcentajes. Y el método utilizado en los antimicrobianos endovenosos que requieren de más de 30 minutos de administración fue bomba jeringa en un 100\% de los casos, lo cual es un aspecto destacable considerando que la literatura no menciona el equipo de administración de fármaco (Tabla 2).

Finalmente, un 92.8\% de los antimicrobianos no se administra con una monitorización específica para cada uno, y en un 7.2\% (vancomicina) se realiza monitorización a través de análisis de laboratorio. En relación con los protocolos locales, no se indica la monitorización específica para cada medicamento. En cambio, en neofax ${ }^{10}$, el 100\% de los antimicrobianos indica algún tipo de monitorización. 


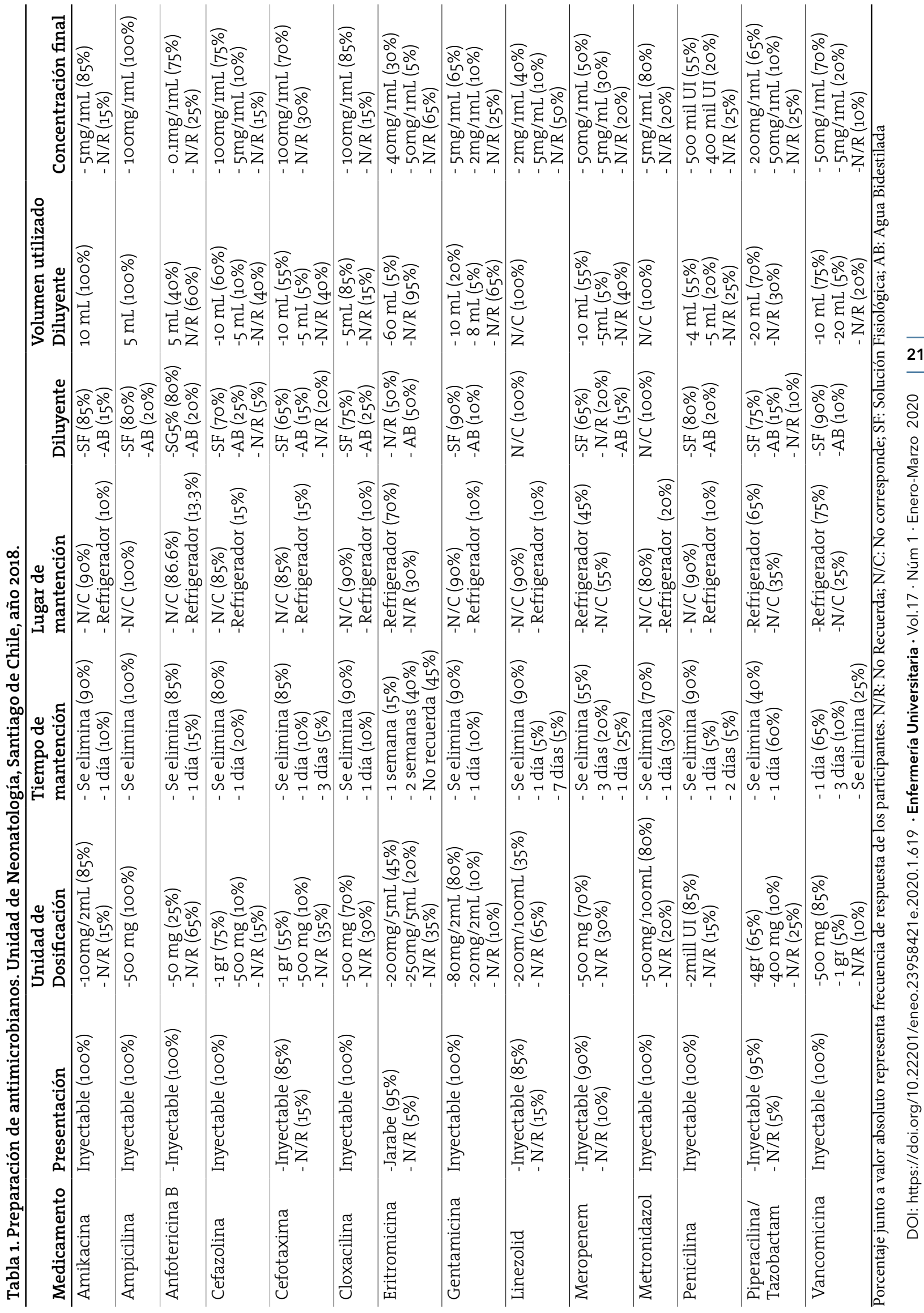


Tabla 2. Administración de Antimicrobianos. Unidad de Neonatología, Santiago de Chile, año 2018

\begin{tabular}{|c|c|c|c|c|}
\hline Medicamento & $\begin{array}{l}\text { Vía de } \\
\text { administración }\end{array}$ & $\begin{array}{l}\text { Tiempo de } \\
\text { administración }\end{array}$ & $\begin{array}{l}\text { Método de } \\
\text { administración }\end{array}$ & Monitorización \\
\hline Amikacina & $\begin{array}{l}\text { Endovenosa } \\
(100 \%)\end{array}$ & 1 Hora $(100 \%)$ & Bomba jeringa (100\%) & $\begin{array}{l}\text { - No (95\%) } \\
\text { - Signos Clínicos (5\%) }\end{array}$ \\
\hline Ampicilina & $\begin{array}{l}\text { Endovenosa } \\
(100 \%)\end{array}$ & $<5 \min (100 \%)$ & $\mathrm{N} / \mathrm{C}(100 \%)$ & $\begin{array}{l}\text { - No (95\%) } \\
\text { - Signos Clínicos (5\%) }\end{array}$ \\
\hline Anfotericina B & $\begin{array}{l}\text { Endovenosa } \\
(100 \%)\end{array}$ & 4-6 Horas $(100 \%)$ & Bomba jeringa (100\%) & $\begin{array}{l}\text { - No (95\%) } \\
\text { - A. de Lab. (5\%) }\end{array}$ \\
\hline Cefazolina & $\begin{array}{l}\text { - Endovenosa } \\
(95 \%) \\
\text { - N/R (5\%) }\end{array}$ & $\begin{array}{l}-1 \text { hora }(90 \%) \\
-30 \min (5 \%) \\
- \text { N/R }(5 \%)\end{array}$ & $\begin{array}{l}\text { - Bomba jeringa (95\%) } \\
\text { - N/R }(5 \%)\end{array}$ & $\begin{array}{l}\text { - No (95\%) } \\
\text { - Signos Clínicos (5\%) }\end{array}$ \\
\hline Cefotaxima & $\begin{array}{l}\text { - Endovenosa } \\
(80 \%) \\
\text { - N/R (20\%) }\end{array}$ & $\begin{array}{l}-1 \text { hora }(80 \%) \\
- \text { N/R }(20 \%)\end{array}$ & $\begin{array}{l}\text { - Bomba jeringa (80\%) } \\
\text { - N/R (20\%) }\end{array}$ & $\begin{array}{l}\text { - No (95\%) } \\
\text { - Signos Clínicos (5\%) }\end{array}$ \\
\hline Cloxacilina & $\begin{array}{l}\text { Endovenosa } \\
(100 \%)\end{array}$ & $<5 \min (100 \%)$ & N/C (100\%) & $\begin{array}{l}\text { - No (95\%) } \\
\text { - Signos Clínicos (5\%) }\end{array}$ \\
\hline Eritromicina & $\begin{array}{l}\text { - Oral }(95 \%) \\
\text { - N/R }(5 \%)\end{array}$ & N/C (100\%) & N/C (100\%) & No (100\%) \\
\hline Gentamicina & $\begin{array}{l}\text { Endovenosa } \\
(100 \%)\end{array}$ & $\begin{array}{l}-1 \text { hora }(95 \%) \\
-30 \min (5 \%)\end{array}$ & Bomba jeringa (100\%) & $\begin{array}{l}\text { - No }(95 \%) \\
\text { - Signos Clínicos (5\%) }\end{array}$ \\
\hline Linezolid & $\begin{array}{l}\text { - Endovenosa } \\
(60 \%) \\
\text { - N/R (40\%) }\end{array}$ & $\begin{array}{l}-1 \text { hora }(75 \%) \\
- \text { N/R }(25 \%)\end{array}$ & $\begin{array}{l}\text { - Bomba jeringa (70\%) } \\
\text { - N/R (30\%) }\end{array}$ & $\begin{array}{l}\text { - No (95\%) } \\
\text { - Signos Clínicos (5\%) }\end{array}$ \\
\hline Meropenem & $\begin{array}{l}\text { - Endovenosa } \\
(90 \%) \\
\text { - N/R (10\%) }\end{array}$ & $\begin{array}{l}-1 \text { hora }(85 \%) \\
- \text { N/R }(15 \%)\end{array}$ & $\begin{array}{l}\text { - Bomba jeringa (90\%) } \\
\text { - N/R (10\%) }\end{array}$ & $\begin{array}{l}\text { - No (95\%) } \\
\text { - Signos Clínicos (5\%) }\end{array}$ \\
\hline Metronidazol & $\begin{array}{l}\text { - Endovenosa } \\
(100 \%)\end{array}$ & $\begin{array}{l}-1 \text { Hora }(95 \%) \\
-30 \min (5 \%) \\
\end{array}$ & Bomba jeringa (100\%) & $\begin{array}{l}\text { - No (95\%) } \\
\text { - Signos Clínicos (5\%) }\end{array}$ \\
\hline Penicilina & $\begin{array}{l}\text { - Endovenosa } \\
(100 \%)\end{array}$ & $\begin{array}{l}-<5 \min (95 \%) \\
-N / R(5 \%) \\
\end{array}$ & N/C (100\%) & No $(100 \%)$ \\
\hline $\begin{array}{l}\text { Piperacilina/ } \\
\text { tazobactam }\end{array}$ & $\begin{array}{l}\text { - Endovenosa } \\
(90 \%) \\
-\mathrm{N} / \mathrm{R}(10 \%)\end{array}$ & $\begin{array}{l}-1 \text { hora }(90 \%) \\
- \text { N/R }(10 \%)\end{array}$ & $\begin{array}{l}\text { - Bomba jeringa (90\%) } \\
\text { - N/R (\%) }\end{array}$ & No $(100 \%)$ \\
\hline Vancomicina & $\begin{array}{l}\text { Endovenosa } \\
(100 \%)\end{array}$ & 3 Horas $(100 \%)$ & Bomba jeringa (100\%) & A. de Lab.(100\%) \\
\hline
\end{tabular}

Nota: N/R: No Recuerda; N/C: No Corresponde; A. de Lab: Análisis de laboratorio

\section{DISCUSIÓN}

Respecto a la variable presentación, es factible indicar que los resultados obtenidos en este trabajo coinciden con lo señalado en la literatura ${ }^{13}$, en cuanto a que la mayoría de los antimicrobianos son inyectables (92.8\%), debido a que los recién nacidos que son hospitalizados en unidades de cuidados neonatales poseen patologías complejas que conllevan tratamientos invasivos como administración de antimicrobianos de este tipo, no es extraño encontrar un alto porcentaje de antimicrobianos que tengan esta presentación.

La unidad de dosificación obtenida en los resultados es la misma que en el manual Neofax ${ }^{10}$ en un $78.5 \%$, sin embargo, en algunos antimicrobianos varía, como amikacina, gentamicina y penicilina, esto probablemente debido a la dosificación que dan los diferentes laboratorios en cada país, a la disponibilidad local de la presentación del fármaco y a la solicitud médica correspondiente. Lo que se aprecia en este caso, es que a pesar de que los profesionales no reconozcan o recuerden todas las unidades de dosificación de los antimicrobianos, esto no tiene consecuencias en el resto del proceso, ya que conocen la dilución y las concentraciones máximas que se deben alcanzar. 
Según los resultados, el diluyente mayormente utilizado es la solución fisiológica, y como segunda opción agua bidestilada, ambas soluciones son compatibles a las indicadas en el manual ${ }^{10}$. En Neofax ${ }^{10}$ sólo en un $28.5 \%$ de estos antimicrobianos se indica la cantidad de diluyente a utilizar, el resto indica utilizar un volumen necesario de diluyente hasta lograr la concentración final, similar a lo recomendado en las normativas locales y a recomendaciones internacionales donde sólo en el $35.7 \%$ se indica el volumen del diluyente a utilizar. Las concentraciones finales luego de la reconstitución son las mismas, al compararlas con el texto de referencia. El cloruro de sodio $0.9 \%$ es uno de los diluyentes más utilizados para la preparación de antimicrobianos, esto debido al aporte de iones, lo que es fundamental para mantener una adecuada homeostasis y mantención de funciones vitales, considerando las pérdidas de agua y electrolitos que sufren los recién nacidos durante los primeros días de vida ${ }^{14}$, es que lo hace un diluyente de primera elección en estas unidades. Además, se ha investigado que los antimicrobianos una vez reconstituidos con este diluyente, sufren sólo una pequeña variación en el pH, con lo que se disminuyen así las complicaciones derivadas de la administración de soluciones más concentradas ${ }^{15}$. Este hallazgo permitiría mantener la recomendación de uso de la solución fisiológica como primera alternativa de diluyente para este tipo de antimicrobianos.

Respecto a la mantención de los medicamentos estudiados, se observaron resultados que son concordantes con lo que indica la literatura del mismo centro, donde se recomienda una estabilidad máxima de 1 día a temperatura ambiente, o máximo 5 días en refrigerador, siendo lo más común hasta 72 horas, se excluyen antimicrobianos que no deben ser refrigerados como linezolid y metronidazol.

Mayoritariamente los profesionales no almacenaban los antimicrobianos una vez reconstituidos, lo que podría ser contradictorio a lo indicado en Neofax 2017, respecto a algunos antimicrobianos, donde se indica mantención del medicamento una vez reconstituido en un 57.1\% de los antimicrobianos estudiados, incluso con una refrigeración de hasta 7010 días con el propósito de reducir los costos asociados a la asistencia médica. Esta medida adoptada por algunos profesionales podría ser justificada como una forma de prevención de infecciones asociadas a la preparación de antimicrobianos, en concordancia a lo que exponen estudios que indican que la mayor causa de infecciones en unidades neonatales está dada por las acciones asociadas a la atención en salud ${ }^{16,17}$.

Revisiones recientes reportan tasas de infección asociadas a prácticas de inyección de medicamentos en 35 por cada 100000 pacientes ${ }^{18}$, por lo que la eliminación de los medicamentos una vez reconstituidos sería válida para disminuir los riesgos de infección. En el caso de los medicamentos que indican mantención, los resultados de la literatura son variables, pudiendo mantenerse desde unas horas a temperatura ambiente, a días en el interior del refrigerador ${ }^{10}$. La mayor diferencia entre los resultados obtenidos y la literatura se da en la variable tiempo de mantención del fármaco vancomicina, donde se observó que en el hospital se mantenía el medicamento almacenado por máximo 24 horas tras su dilución; en cambio, en la norma mencionada, se recomienda mantener máximo por 14 días ${ }^{10}$. A su vez, la Guía de Administración de Fármacos en el Periodo Neonatal para enfermería ${ }^{19}$, recomienda no mantener ningún fármaco por más de 24 horas luego de la reconstitución y dilución, para evitar problemas de inestabilidad físico-química y microbiológica, lo cual sería concordante con la información recolectada en esta investigación.

Los tiempos de administración varían al realizar la comparación con la literatura. Los encuestados refieren que lo más utilizado era administrar antimicrobianos durante 1 hora mediante bomba de infusión continua, a excepción de los que pueden administrarse en bolo (administración de un medicamento en menos de 5 minutos), y los que requieren de un período más largo como la 
anfotericina B y la vancomicina. La administración por tiempo de infusión prolongado está justificada, para evitar complicaciones como hipervolemia, insuficiencia cardíaca y toxicidad ${ }^{15}$, ya que algunos neonatos requieren la perfusión de más de una solución. Por lo expuesto, sería adecuado mantener estándares de administración que consideren al menos 1 hora con uso de bomba de infusión.

En cuanto a la monitorización hay diferencias significativas entre los resultados y la literatura; en Neofax ${ }^{10}$, la mayoría de los fármacos requiere algún tipo de monitorización, esto orientado a los efectos secundarios de cada medicamento y/o a las patologías o alteraciones del paciente; de acuerdo con esto, pueden solicitarse exámenes de laboratorio como concentraciones plasmáticas, hemograma, electrolitos, enzimas hepáticas, etc. Además, podría requerir alguna monitorización clínica específica o la monitorización seriada de algún signo vital. Según los resultados obtenidos, el único medicamento en que todos realizan monitorización según lo que se recomienda, es la vancomicina.

La literatura menciona la importancia de realizar monitorización, ya que este proceso influye en las decisiones clínicas, en especial en recién nacidos, donde interfieren varios factores relacionados con su fisiología como variabilidad farmacocinética ${ }^{20}$, la maduración de sus sistemas y además las comorbilidades, también relacionados con el fármaco, si se utilizaron dosis de carga o el tiempo de respuesta del medicamento, y con la composición de proteínas plasmáticas, se logra la asociación concentración-eficacia del fármaco o concentración-toxicidad ${ }^{21,22}$. La vigilancia terapéutica debe ser individual y adaptarse a las necesidades de los recién nacido ${ }^{21}$. A pesar de las recomendaciones, en la práctica clínica no todos los antimicrobianos tienen vigilancia mediante niveles plasmáticos; esto se debe a que muchos de los antimicrobianos no tienen estrecho margen terapéutico y la mayoría de los eventos adversos no son dosis dependiente ${ }^{23,24}$. Respecto a la familia de antimicrobianos, en Chile habitualmente se realiza vigilancia de amikacina, gentamicina y vancomicina, por ser útil en disminuir oto y nefrotoxicidad ${ }^{23}$. Por lo anteriormente mencionado, se recomienda incorporar mayor vigilancia en los aminoglucósidos y además realizar nuevos estudios en la unidad para evaluar de manera más exhaustiva la práctica de vigilancia farmacológica, incorporar recomendaciones y con esto ajustar protocolos locales, con el fin de realizar un uso racional y efectivo de los antimicrobianos, y además optimizar los recursos económicos disponibles.

En el caso de los antimicrobianos que se administran vía oral como eritromicina, los profesionales no recuerdan las unidades de dosificación, el tiempo y lugar de mantención, esto debido a que la preparación y administración es delegada a técnicos en enfermería (trabajador del hospital que no tiene un título profesional). No existe registro de administración de este fármaco en los manuales locales, lo que puede incrementar la variabilidad en el proceso de preparación y administración, siendo un riesgo innecesario en estas unidades.

Finalmente, las limitaciones del estudio, en primer lugar, tienen relación con el instrumento, el cual podría requerir una validación más exhaustiva para poder ser utilizado en otras investigaciones. Adicionalmente destaca la existencia de un sesgo de información propio de los participantes, quienes al no preparar de forma frecuente todos los medicamentos, podrían no estar familiarizados con ciertos antimicrobianos. En segundo lugar, el estudio no considera segundas diluciones, lo que es necesario en ciertos antimicrobianos para lograr la concentración final. Por último, limitaciones relacionadas con el espacio-tiempo: la aplicación del instrumento ocurrió mientras los profesionales se encontraban en horario laboral, entre sus funciones clínicas, lo que podría afectar los resultados observados, y además podría ser un factor relevante para que parte del universo no respondiera. En este punto es relevante mencionar que en ningún caso los participantes fueron obligados a 
responder, esto para resguardar el gran compromiso del servicio con la universidad que desarrolló esta investigación.

\section{CONCLUSIONES}

En cuanto al proceso de dilución de los antimicrobianos endovenosos en unidades de neonatología, los resultados destacan la utilización mayoritaria de solución fisiológica y agua bidestilada, siendo ambos diluyentes compatibles con la mayoría de los antimicrobianos, sin provocar efectos adversos. Como recomendación se propone la utilización de solución fisiológica, ya que presenta ventajas en la mantención de propiedades físico-químicas en la preparación de antimicrobianos, con las precauciones de no administrar en exceso.

El tiempo de mantención de antimicrobianos fue una variable que presentó grandes variaciones entre las respuestas de los profesionales. En un mismo fármaco los resultados varían desde la eliminación inmediata del fármaco una vez reconstituido al mantenimiento durante días, siendo más prevalente la primera opción. Debido a la importancia de este proceso, en la mantención de las propiedades farmacológicas y la prevención de infecciones asociadas a la atención en salud (IAAS), es que se recomienda el mejoramiento de protocolos locales y su revisión continua, y tal como recomienda la literatura, no mantener los antimicrobianos por más de 24 horas, además mejorar la difusión y accesibilidad del material.

La monitorización que se realiza al neonato luego de la administración de antimicrobianos es un proceso que no está del todo estandarizado, observándose que en la mayoría de los tratamientos con antimicrobianos no se realiza una vigilancia específica por fármaco. Este aspecto requiere de un análisis mayor para realizar recomendaciones, por lo que se sugiere realizar a futuro nuevas investigaciones enfocadas en este tema, ya que interfieren otras variables que no fueron consultadas en este estudio, como los días de tratamiento, administración de otros antimicrobianos y patologías concomitantes.

A partir de estos resultados se propone la confección o mejora de protocolos de acción frente a la preparación, mantención, administración y monitorización de antimicrobianos en unidades neonatales que estén a disposición de todos los profesionales a través de un medio de difusión simple y facilitador, para la estandarización en procesos; $y$, adicionalmente, agregar variables que no están consideradas en el protocolo local como reconstitución, volúmenes de diluyente para cada fármaco y monitorización, y así resguardar la seguridad de los pacientes y disminuir efectos adversos en la atención.

Como recomendación final, se sugiere realizar nuevas investigaciones enfocadas en la farmacología clínica y de la monitorización o vigilancia farmacológica, pudiendo replicarse este estudio en otros centros hospitalarios o considerar otras familias de antimicrobianos, con el objetivo de mejorar las prácticas clínicas en beneficio de los usuarios.

\section{RESPONSABILIDADES ÉTICAS}

Protección a personas y animales. Los autores declaran que en este estudio no se realizaron experimentos con personas o animales.

Confidencialidad. Los participantes firmaron un consentimiento informado y se les ofreció la seguridad de resguardar sus datos personales.

Financiamiento. Ninguno.

Conflicto de intereses. Los autores declaran no tener conflicto de intereses. 


\section{REFERENCIAS}

1. Agra Varela Y, Aguayo Maldonado J, Álvarez González M, Botet Mussons F, Bustos Lozano G, Díaz Torres $\mathrm{P}$, et al. Unidades de neonatología. Estándares y recomendaciones de calidad. Madrid: Ministerio De Sanidad, Servicios Sociales E Igualdad. 2014. 11 p.

2. Mena P, Mülhausen G, Novoa J, Vicanco G. Guías nacionales de Neonatología. Santiago, Chile: Ministerio de Salud; 2005. p. 467. https://bit.ly/3167dnj

3. Allegaert K, Anker J van den. Neonatal Drug Therapy: The First Frontier of Therapeutics for Children. Clin Pharmacol Ther. 2015; 98(3): 288-97. https://doi.org/10.1002/cpt.166

4. Allegaert K, van de Velde $M$, van den Anker J. Neonatal clinical pharmacology. Pediatr Anesth 2014; 24(1): 30-8. https://doi.org/10.1111/pan.12176

5. Mulugeta Y (Lily), Zajicek A, Barrett J, Sachs HC, McCune S, Sinha V, et al. Development of Drug Therapies for Newborns and Children: The Scientific and Regulatory Imperatives. Pediatr Clin North Am. 2017. 64(6):1185-96. https://doi.org/10.1016/j.pcl.2017.08.015

6. Wang J, Avant D, Green D, Seo S, Fisher J, Mulberg AE, et al. A Survey of Neonatal Pharmacokinetic and Pharmacodynamic Studies in Pediatric Drug Development. Clinl Pharmacol Ther. 2015; 98(3): 328-35. https://doi.org/10.1002/cpt.149

7. Allegaert K, Van Den Anker JN. Clinical pharmacology in neonates: Small size, huge variability. Neonatology. 2014; 105(4): 344-9. https://doi.org/10.1159/000360648

8. Smits A, Kulo A, N de Hoon J, Allegaert K. Pharmacokinetics of Drugs in Neonates: Pattern Recognition Beyond Compound Specific Observations. Curr Pharm Des 2012; 18(21): 3119-46. https://doi.org/10.2174/1381612811209023119

9. Anderson BJ, Allegaert K. The pharmacology of anaesthetics in the neonate. Best Pract Res Clin Anaesthesiol. 2010; 24(3): 419-31. https://doi.org/10.1016/j.bpa.2010.02.019

10. Micromedex Neofax Online. Neofax. 2017. neofax.micromedexsolutions.com

11. Hernández S, Arriagada A, González M. Administración de medicamentos endovenosos en pacientes adultos, pediátricos y neonatales. $5^{\mathrm{a}}$. ed. Santiago, Chile: Servicio de salud metropolitano occidente Hospital San Juan de Dios- CDT; 2010.

12. González J. Almacenamiento y conservación de medicamentos. za ed. Santiago, Chile: Servicio de salud metropolitano occidente Hospital San Juan de Dios- CDT; 2011.

13. Jimeno S, Riaza M, Ortiz-Villajos C, Cuñaro A. Guía Farmacoterapeutica Neonatal. España: Ministerio de Sanidad y Política Social; 2011. 104 p. https://bit.ly/37IEoOQv

14. Jonguitud-Aguilar A, Calvillo-Robles S, Ruiz-Martínez E, Olvera-López G. Protocolo de manejo en deshidratación hipernatrémica neonatal. Perinatol y Reprod Humana. 2015; 29(2): 65-9.

https://doi.org/10.1016/j.rprh.2015.05.003

15. Monteiro C, Crepaldi RMC, Avelar AFM, Peterlini MAS, Pedreira MDLG. Potencial hidrogeniônico de soluções de antibióticos submetidas a condições ambientais: Ensaio preliminar. Rev Esc Enferm USP. 2012; 46(2): 311-9. http://dx.doi.org/10.1590/Soo80-62342012000200007

16. Urzúa Baquedano S, Ferrés M, García P, Sánchez A, Luco M. Estrategias para reducir infecciones, uso de antimicrobianos y sus efectos en una unidad de neonatología. Rev Chil Infectol. 2017; 34(2): 99-107. http://dx.doi.org/10.4067/So716-10182017000200001

17. Chakkarapani AA, Russell AB. Antibiotic stewardship in the neonatal intensive care unit. Paediatr Child Health (Oxford) 2019; 29(6): 269-73. https://doi.org/10.1016/j.paed.2019.03.004

18. Curran E. Intravenous drug preparation: The infection risks. Br J Nurs. 2011; 2O(14): S4-S6. https://doi.org/10.12968/bjon.2011.20.Sup7.S4 
19. Quesada A, López O, Jiménez E, Cabañas M. Guía de administración de fármacos en el periodo neonatal para enfermería. 2a. ed. Barcelona, España: Elsevier; 2015. 88 p. https://bit.ly/318Vd4n

20. Samardzic J, Allegaert K, Wilbaux M, Pfister M, van den Anker JN. Quantitative clinical pharmacology practice for optimal use of antibiotics during the neonatal period. Expert Opin Drug Metab Toxicol. 2016; 12(4): 367-75. http://dx.doi.org/10.1517/17425255.2016.1147559

21. Pauwels S, Allegaert K. Therapeutic drug monitoring in neonates. Arch Dis Child 2016;101(4):377-81 https://doi.org/10.1136/archdischild-2013-305309

22. Rodieux F, Wilbaux M, van den Anker JN, Pfister M. Effect of Kidney Function on Drug Kinetics and Dosing in Neonates, Infants, and Children. Clin Pharmacokinet 2015; 54(12): 1183-204. https://doi.org/10.1007/s40262-015-0298-7

23. Escobar L. Monitorización terapéutica de fármacos y aspectos prácticos de farmacocinética. Rev Médica Clínica Las Condes 2016; 27(5): 605-14. Disponible en: https://doi.org/10.1016/j.rmclc.2016.09.006

24. Palavecino $M$. Toxicidad antibacterianos: farmacocinética-farmacodinamia: prevención y manejo. Rev Médica Clínica Las Condes. 2014; 25(3):445-56.https://doi.org/10.1016/So716-8640(14)70o61-6 